\title{
CESÀRO MEANS OF FOURIER SERIES ON ROTATION GROUPS
}

\author{
DASHAN FAN
}

(Communicated by J. Marshall Ash)

\begin{abstract}
We study the Cesàro means of Fourier series on rotation groups $\mathrm{SO}(3)$ and $\mathrm{SO}(4)$. On these two classical groups, we solve an open question recently posted in Harmonic analysis on classical groups [Springer-Verlag, Berlin, and Science Press, Beijing, 1991].
\end{abstract}

Let $\mathrm{SO}(n)$ be the rotation group on $\mathbb{R}^{n}$. By $[\mathrm{H}]$, it is known that one can identify $\mathrm{SO}(n)$ as the characteristic manifold of the classical domain $\mathscr{R}_{n}$ which was studied by E. Cartan (see [C] for the definition of $\mathscr{R}_{n}$ ). To solve the Dirichlet problem on $\mathscr{R}_{n}$, Hua proved, from the view of several complex variables, that the Poisson kernel on $\mathscr{R}_{n}$ is (see $[\mathrm{H}]$ )

$$
P\left(X_{0}, \Gamma\right)=\frac{\operatorname{det}\left(I-X_{0} X_{0}^{\prime}\right)^{(n-1) / 2}}{\operatorname{det}\left(I-X_{0} \Gamma^{\prime}\right)^{n-1}},
$$

where $I$ is the identity element in $\mathrm{SO}(n)$ and $X^{\prime}$ is the transpose of a matrix $X$.

From the above explicit formula of the Poisson kernel, Gong defined the Cesàro kernel on $\mathrm{SO}(n)$ as follows (see [G, p. 140]).

Let $d V$ be the normalized Haar measure of $\mathrm{SO}(n)$. For $\alpha>-1$ and any positive integer $N$, let $A_{N}^{\alpha}=(\alpha+N) \cdots(\alpha+1) / N$ !. Then the Cesàro kernel $K_{N}^{\alpha}(V)$ on $\mathrm{SO}(n)$ is defined by

$$
K_{N}^{\alpha}(V)=\operatorname{det}^{(n-1) / 2}\left(\left\{A_{N}^{\alpha} I+\sum_{j=1}^{N}\left(V^{j}+V^{\prime j}\right) \sum_{\nu=0}^{N-j} A_{\nu}^{\alpha-1}\right\} / A_{N}^{\alpha}\right) / B_{N}^{\alpha},
$$

where

(3) $\quad B_{N}^{\alpha}=\int_{\mathrm{SO}(n)} \operatorname{det}^{(n-1) / 2}\left(\left\{A_{N}^{\alpha} I+\sum_{j=1}^{N}\left(V^{j}+V^{\prime j}\right) \sum_{\nu=0}^{N-j} A_{\nu}^{\alpha-1}\right\} / A_{N}^{\alpha}\right) d V$.

We easily see, from the above definition, that for any integer $N$ and any $V \in$ $\mathrm{SO}(n)$,

$$
\int_{\mathrm{SO}(n)} K_{N}^{\alpha}(U V) d U=1
$$

Received by the editors January 8, 1993 and, in revised form, June 29, 1993.

1991 Mathematics Subject Classification. Primary 43A55, 43A50, 43A80; Secondary 22E20.

Key words and phrases. Fourier series, Cesàro means, rotation groups, Lebesgue constants. 
Because $\mathrm{SO}(2)$ can be identified with the one-dimensional torus $\mathbb{T}$, it is also easy to see that the above definition (2) is the classical $(c, \alpha)$ kernel of the Fourier series on $\mathbb{T}$ (see $[\mathrm{Z}]$ ) when $n=2$.

In [G], Gong proved the following convergence theorem on $\mathrm{SO}(n)$ :

Theorem A. Suppose that $f$ is any continuous function on $\mathrm{SO}(n)$. If the index $\alpha$ is greater than $(n-2) /(n-1)$, then

$$
\lim _{N \rightarrow \infty}\left(K_{N}^{\alpha} * f\right)(V)=f(V) .
$$

It is a well-known fact that (see [Z]) in Theorem A the condition $\alpha>$ $(n-2) /(n-1)$ is sharp for $n=2$. Thus, Gong posed an open question: whether the condition $\alpha>(n-2) /(n-1)$ can be improved when $n \geq 3$ ?

In this note, we solve the above question on $\mathrm{SO}(3)$ and $\mathrm{SO}(4)$. The main result consists of the following two theorems.

Theorem 1 (Result on $\mathrm{SO}(3)$ ). (i) Suppose that $f$ is a continuous function on $\mathrm{SO}(3)$. If $\alpha_{0}=\frac{1}{2}$, then for any $V \in \mathrm{SO}(3), \lim _{N \rightarrow \infty}\left(K_{N}^{\alpha_{0}} * f\right)(V)=f(V)$.

(ii) For any $\alpha \in\left(-1, \frac{1}{2}\right)$ there is a $C^{\infty}$ function $g(V)$ on $\mathrm{SO}(3)$ such that

$$
\varlimsup_{N \rightarrow \infty}\left(K_{N}^{\alpha} * g\right)(I) \neq g(I) .
$$

Theorem 2 (Result on $\mathrm{SO}(4)$ ). (i) Let $\alpha_{0}=\frac{2}{3}$; then

$$
\int_{\mathrm{SO}(4)}\left|K_{N}^{\alpha_{0}}(V)\right| d V \geq A \log N \quad \text { as } N \rightarrow \infty .
$$

(ii) For $\alpha \in(-1,0)$,

$$
\int_{\mathrm{SO}(4)}\left|K_{N}^{\alpha}(V)\right| d V \geq A N \quad \text { as } N \rightarrow \infty .
$$

(iii) For $\alpha \in\left(\frac{1}{2}, \frac{2}{3}\right)$,

$$
\int_{\mathrm{SO}(4)}\left|K_{N}^{\alpha}(V)\right| d V \geq A N^{2-3 \alpha} \quad \text { as } N \rightarrow \infty .
$$

(iv) For $\alpha \in\left[0, \frac{1}{2}\right)$,

$$
\int_{\mathrm{SO}(4)}\left|K_{N}^{\alpha}(V)\right| d V \geq A N^{1-\alpha} \text { as } N \rightarrow \infty .
$$

(v)

$$
\int_{\mathrm{SO}(4)}\left|K_{N}^{1 / 2}(V)\right| d V \geq A N^{1 / 2} / \log N \quad \text { as } N \rightarrow \infty .
$$

In the above formulas, $A$ is a constant independent of $N$.

Notes. By the well-known Banach-Steinhaus theorem, Theorem 2 implies that Theorem A fails on $\mathrm{SO}(4)$ if $\alpha \in\left(-1, \frac{2}{3}\right]$.

Before proving these two theorems, we need to derive a more explicit formula of the kernel $K_{N}^{\alpha}(V)$.

Let $S(\theta)$ be the $2 \times 2$ matrix

$$
\left(\begin{array}{cc}
\cos \theta & \sin \theta \\
-\sin \theta & \cos \theta
\end{array}\right)
$$


and $C(\theta)$ be the $2 \times 2$ matrix

$$
\left(\begin{array}{cc}
\cos \theta & 0 \\
0 & \cos \theta
\end{array}\right)
$$

Recall that any $V \in \mathrm{SO}(2 k)$ is conjugate to a $2 k \times 2 k$ matrix $T(\theta)$ which is $S\left(\theta_{1}\right) \oplus S\left(\theta_{2}\right) \oplus \cdots \oplus S\left(\theta_{k}\right)$ and that any $V \in \mathrm{SO}(2 k+1)$ is conjugate to a $(2 k+1) \times(2 k+1)$ matrix $T(\theta)$ which equals $S\left(\theta_{1}\right) \oplus S\left(\theta_{2}\right) \oplus \cdots \oplus S\left(\theta_{k}\right) \oplus 1$, where $\left(\theta_{1}, \theta_{2}, \ldots, \theta_{k}\right)$ is a coordinate satisfying

$$
-\pi \leq \theta_{j} \leq \pi, \quad j=1,2, \ldots, k .
$$

Noticing that $\sum_{\nu=0}^{N-j} A_{\nu}^{\alpha-1}=A_{n-j}^{\alpha}$ (see [Z, p. 77]) and that the determinant is a central function, we easily see that

$$
K_{N}^{\alpha}(V)=\operatorname{det}^{(n-1) / 2}\left(\left\{A_{N}^{\alpha} I+\sum_{j=1}^{N}\left(T(\theta)^{j}+T(\theta)^{\prime j}\right) A_{N-j}^{\alpha}\right\} / A_{N}\right) / B_{N}^{\alpha} .
$$

Using induction, we also easily obtain that

$$
T(\theta)^{j}=T(j \theta) \quad \text { and } \quad T(\theta)^{\prime j}=T(j \theta)^{\prime} .
$$

Thus by the definition of $T(\theta)$, if $n=2 k$, then

$$
T(\theta)^{j}+T(\theta)^{\prime j}=2^{k} C\left(j \theta_{1}\right) \oplus C\left(j \theta_{2}\right) \oplus \cdots \oplus C\left(j \theta_{k}\right) .
$$

In this case we obtain that

$$
\left\{A_{N}^{\alpha} I+\sum_{j=1}^{N}\left(T(\theta)^{j}+T(\theta)^{\prime j}\right) A_{N-j}^{\alpha}\right\} / A_{N}^{\alpha}
$$

is a $2 k \times 2 k$ matrix:

$$
\sigma_{N}^{\alpha}\left(\theta_{1}\right) \oplus \sigma_{N}^{\alpha}\left(\theta_{1}\right) \oplus \sigma_{N}^{\alpha}\left(\theta_{2}\right) \oplus \sigma_{N}^{\alpha}\left(\theta_{2}\right) \oplus \cdots \oplus \sigma_{N}^{\alpha}\left(\theta_{k}\right) \oplus \sigma_{N}^{\alpha}\left(\theta_{k}\right),
$$

where $\sigma_{N}^{\alpha}(\theta)=\frac{1}{2}+\sum_{j=1}^{N} \cos j \theta A_{N-j}^{\alpha} / A_{N}^{\alpha}$ is the one-dimensional Cesàro kernel (see $[Z, 1.14$, p. 77 and 5.2, p. 94]). Therefore, by noticing the definition of $B_{N}^{\alpha}$, we clearly see that the Cesàro kernel on $\mathrm{SO}(2 k)$ is

$$
K_{N}^{\alpha}(V)=\prod_{j=1}^{k}\left\{\sigma_{N}^{\alpha}\left(\theta_{j}\right)\right\}^{2 k-1} / \tilde{B}_{N}^{\alpha}
$$

where

$$
\tilde{B}_{N}^{\alpha}=\int_{-\pi \leq \theta_{k} \leq \cdots \leq \theta_{1} \leq \pi} \ldots \int_{j=1}^{k}\left\{\sigma_{N}^{\alpha}\left(\theta_{j}\right)\right\}^{2 k-1} \prod_{1 \leq i<j \leq k}\left(\cos \theta_{i}-\cos \theta_{j}\right)^{2} d \theta_{1} \cdots d \theta_{k}
$$

and $\prod_{1 \leq i<j \leq k}\left(\cos \theta_{i}-\cos \theta_{j}\right)^{2}$ is the Weyl function, up to a constant multiplier, on $\mathrm{SO}(\overline{2} k)$ (see $[\mathrm{W}])$.

If $n=2 k+1$, then

$$
T(\theta)^{j}+T(\theta)^{\prime j}=2^{k+1} C\left(j \theta_{1}\right) \oplus C\left(j \theta_{2}\right) \oplus \cdots \oplus C\left(j \theta_{k}\right) \oplus 1 .
$$


Similar to the case of $n=2 k$, we easily see that

$$
\begin{aligned}
A_{N}^{\alpha} I & +\sum_{j=1}^{N}\left(T(\theta)^{j}+T(\theta)^{\prime j}\right) A_{N-j}^{\alpha} / A_{N}^{\alpha} \\
& =\sigma_{N}^{\alpha}\left(\theta_{1}\right) \oplus \sigma_{N}^{\alpha}\left(\theta_{1}\right) \oplus \cdots \oplus \sigma_{N}^{\alpha}\left(\theta_{k}\right) \oplus \sigma_{N}^{\alpha}\left(\theta_{k}\right) \oplus(2 N+1) .
\end{aligned}
$$

Thus the Cesàro kernel on $\mathrm{SO}(2 k+1)$ is

$$
K_{N}^{\alpha}(V)=\prod_{j=1}^{k}\left\{\sigma_{N}^{\alpha}\left(\theta_{j}\right)\right\}^{2 k} / \tilde{B}_{N}^{\alpha},
$$

where

$$
\begin{aligned}
\tilde{B}_{N}^{\alpha}= & \int_{-\pi \leq \theta_{k} \leq \cdots \leq \theta_{1} \leq \pi} \ldots \int_{j=1} \prod_{N}^{k}\left\{\sigma_{N}^{\alpha}\left(\theta_{j}\right)\right\}^{2 k} \\
& \times\left(1-\cos \theta_{j}\right) \prod_{1 \leq i<j \leq k}\left(\cos \theta_{i}-\cos \theta_{j}\right)^{2} d \theta_{1} \cdots d \theta_{k}
\end{aligned}
$$

and

$$
\prod_{j=1}^{k}\left(1-\cos \theta_{j}\right) \prod_{1 \leq i<j \leq k}\left(\cos \theta_{i}-\cos \theta_{j}\right)
$$

is the Weyl function, up to a constant multiplier, on $\mathrm{SO}(2 k+1)$ (see [W]).

Recall the following estimates of the classical Cesàro kernels:

Lemma 1. If $\alpha>-1$ and $|\theta| \leq N^{-1}$, then $B N \geq \sigma_{N}^{\alpha}(\theta) \geq A N$, where $A, B$ are positive constants independent of $\theta$ and $N$. If $\alpha>-1$ and $|\theta|>N^{-1}$, then

$$
\begin{aligned}
\left\{\sigma_{N}^{\alpha}(\theta)\right\}^{n}= & \left.\sin ^{n}\{(N+(1+\alpha) / 2) \theta-\pi \alpha / 2\} /(\alpha)_{N}^{n}(2 \sin (\theta / 2))^{n(\alpha+1)}\right\} \\
& +O\left(N^{-(n-1) \alpha-1} \theta^{-(n-1)(\alpha+1)-2}\right),
\end{aligned}
$$

where $(\alpha)_{N}=\Gamma(\alpha+N+1) /\{\Gamma(\alpha+1) \Gamma(N+1)\} \cong N^{\alpha}$ for $N$ sufficiently large.

Proof. See [Z, pp. 77, 95] for the proof.

Now we are ready to prove the main theorems.

Proof of Theorem 1. Let $d(U, I)$ be the Euclidean distance between $U$ and $I$; then $d(U, I)=2^{1 / 2}(1-\cos \theta)^{1 / 2}$, where $U$ is conjugate to the element $S(\theta) \oplus 1$ (see [G, p. 153]). We denote the modulus of continuity of a continuous function $f$ by $\omega(f ; \delta)$. Then by noticing formula $(4)$ and that $K_{N}^{\alpha}(V)$ is a positive kernel on $\mathrm{SO}(2 k+1)$, we have

$$
\begin{aligned}
\left|\left(K_{N}^{\alpha_{0}} * f\right)(V)-f(V)\right| \\
\quad=\left|\int_{\mathrm{SO}(3)} K_{N}^{\alpha_{0}}(U)\left\{f\left(U^{-1} V\right)-f(V)\right\} d U\right| \\
\quad \leq \omega(f ; 2 \delta) \int_{|\theta| \leq \delta} K_{N}^{\alpha_{0}}(U) d U+2\|f\|_{\infty} \int_{|\theta| \geq \delta} K_{N}^{\alpha_{0}}(U) d U \\
\quad \leq \omega(f ; 2 \delta)+2\|f\|_{\infty} \int_{|\theta| \geq \delta} K_{N}^{\alpha_{0}}(U) d U
\end{aligned}
$$


Notice that $\omega(f ; 2 \delta)$ goes to zero as $\delta$ tends to zero. Thus to prove (i) in Theorem 1 , it suffices to show that for any fixed $\delta>0$,

$$
\lim _{N \rightarrow \infty} \int_{|\theta| \geq \delta} K_{N}^{\alpha_{0}}(U) d U=0 .
$$

By (10), we know that

$$
\int_{|\theta| \geq \delta} K_{N}^{\alpha_{0}}(U) d U=\int_{|\theta| \geq \delta}\left\{\sigma_{N}^{\alpha_{0}}(\theta)\right\}^{2}(1-\cos \theta) d \theta / \widetilde{B}_{N}^{\alpha_{0}}
$$

where

$$
\widetilde{B}_{N}^{\alpha_{0}}=\int_{-\pi}^{\pi}\left\{\sigma_{N}^{\alpha_{0}}(\theta)\right\}^{2}(1-\cos \theta) d \theta .
$$

By the definition of the Cesàro kernel together with (11), one easily sees that

$$
\begin{aligned}
\int_{|\theta| \geq \delta} & \left\{\sigma_{N}^{\alpha_{0}}(\theta)\right\}^{2}(1-\cos \theta) d \theta \\
& \leq N^{-1} \int_{|\theta| \geq \delta}|\theta|^{-3}(1-\cos \theta) d \theta \leq A_{\delta} N^{-1},
\end{aligned}
$$

where $A_{\delta}$ is a constant depending only on $\delta$.

On the other side, by (11) we have

$$
\begin{aligned}
\widetilde{B}_{N}^{\alpha_{0}} \geq & N^{-1} \int_{1 / N}^{\pi} \sin ^{2}\left\{\left(N+\frac{3}{4}\right) \theta-\pi / 4\right\} \sin ^{-3}(\theta / 2)(1-\cos \theta) d \theta \\
& +O\left(N^{-3 / 2} \int_{1 / N}^{\pi} \theta^{-7 / 2}(1-\cos \theta) d \theta\right) \\
\geq & N^{-1} \int_{1 / N}^{\pi} \sin ^{2}\left\{\left(N+\frac{3}{4}\right) \theta-\pi / 4\right\} \theta^{-1} d \theta+O\left(N^{-1}\right)
\end{aligned}
$$

This shows that

$$
\widetilde{B}_{N}^{\alpha_{0}} \geq A N^{-1} \log N \quad(N \rightarrow \infty) .
$$

Equations (14) and (15) furnish the proof of (i) in Theorem 1.

Next let $g(U)=(1-\cos \theta)$; then $g(I)=0$. We want to prove that this $C^{\infty}$ function $g(U)$ furnishes the second part of Theorem 1. In fact,

$$
\left(K_{N}^{\alpha} * g\right)(I)-g(I)=\int_{\mathrm{SO}(3)} K_{N}^{\alpha}(U) g(U) d U=I_{N}^{\alpha} / \widetilde{B}_{N}^{\alpha},
$$

where

$$
\begin{gathered}
I_{N}^{\alpha}=\int_{-\pi}^{\pi}\left\{\sigma_{N}^{\alpha}(\theta)\right\}^{2}(1-\cos \theta)^{2} d \theta, \\
\widetilde{B}_{N}^{\alpha}=\int_{-\pi}^{\pi}\left\{\sigma_{N}^{\alpha}(\theta)\right\}^{2}(1-\cos \theta) d \theta .
\end{gathered}
$$

Noticing that $\alpha \in\left(-1, \frac{1}{2}\right)$, by Lemma 1 we have (16) $\widetilde{B}_{N}^{\alpha}=O(1 / N)+O\left(N^{-2 \alpha} \int_{1 / N}^{\pi} \theta^{-2(\alpha+1)+2} d \theta\right)=O\left(N^{-2 \alpha}\right) \quad$ as $N \rightarrow \infty$. 
Using formula (11) and noticing $\alpha \in\left(-1, \frac{1}{2}\right)$, we obtain that

$$
\begin{aligned}
I_{N}^{\alpha} \geq & \left.A N^{-2 \alpha} \int_{1 / N}^{\pi} \sin ^{2}\{(N+(1+\alpha) / 2) \theta-\pi \alpha / 2)\right\}(1-\cos \theta)^{2} \sin ^{-2(\alpha+1)}(\theta / 2) d \theta \\
& +O\left(N^{-\alpha-1} \int_{1 / N}^{\pi} \theta^{-\alpha-3}(1-\cos \theta)^{2} d \theta\right) .
\end{aligned}
$$

Thus, an easy computation shows that

$$
I_{N}^{\alpha} \geq A N^{-2 \alpha} \quad(N \rightarrow \infty) .
$$

From (16) and (17), we know that

$$
\varlimsup_{N \rightarrow \infty} \int_{\mathrm{SO}(3)} K_{N}^{\alpha}(U) g(U) d U>0=g(I) .
$$

Theorem 1 is proved.

Proof of Theorem 2. Let $\alpha \in\left(-1, \frac{2}{3}\right]$. We need to calculate the Lebesgue constant $K_{N}^{\alpha}(V)$. By formula (8), we know that $\int_{\mathrm{SO}(4)}\left|K_{N}^{\alpha}(V)\right| d V$ is equal to

$$
\iint_{-\pi \leq \theta_{2}<\theta_{1} \leq \pi}\left|\sigma_{N}^{\alpha}\left(\theta_{1}\right) \sigma_{N}^{\alpha}\left(\theta_{2}\right)\right|^{3}\left(\cos \theta_{1}-\cos \theta_{2}\right)^{2} d \theta_{1} d \theta_{2} / \widetilde{B}_{N}^{\alpha}=J_{N}^{\alpha} / \widetilde{B}_{N}^{\alpha}
$$

By a symmetric argument, $\widetilde{B}_{N}^{\alpha}$ in (18) is equal to

$$
\begin{gathered}
2 \int_{-\pi}^{\pi} \int_{-\pi}^{\pi} \prod_{k=1}^{2}\left\{\sigma_{N}^{\alpha}\left(\theta_{k}\right)\right\}^{3}\left\{\left(1-\cos \theta_{1}\right)-\left(1-\cos \theta_{2}\right)\right\}^{2} d \theta_{1} d \theta_{2} \\
\quad=2\left\{\int_{\substack{k=1 \\
\left|\theta_{1}\right| \leq 1 / N \\
\left|\theta_{2}\right| \leq 1 / N}}+\iint_{\left|\theta_{1}\right| \geq 1 / N}+2 \iint_{\substack{\theta_{2} \mid \geq 1 / N \\
\text { | } \\
\left|\theta_{1}\right| \geq 1 / N \\
\left|\theta_{2}\right| \leq 1 / N}}\right\}=J+J J+J J J .
\end{gathered}
$$

One easily sees that for any $\alpha>-1$,

$$
J=O(1) \quad(N \rightarrow \infty) .
$$

By (11) again, the third term $J J J$ in (19) is dominated by

$$
\begin{aligned}
& O\left(N^{3-3 \alpha} \int_{-1 / N}^{1 / N} \int_{\left|\theta_{1}\right| \geq 1 / N} \sin ^{3}\left\{(N+(1+\alpha) / 2) \theta_{1}-\pi \alpha / 2\right\}\right. \\
& \left.\times\left\{\left|\theta_{1}\right|^{-3(\alpha+1)} \theta_{2}^{4}+\left|\theta_{1}\right|^{-3 \alpha-1} \theta_{2}^{2}+\left|\theta_{1}\right|^{-3 \alpha+1}\right\} d \theta_{1} d \theta_{2}\right) \\
& \quad+O\left(N^{2-2 \alpha} \int_{-1 / N}^{1 / N} \int_{\left|\theta_{1}\right| \geq 1 / N}\left\{\left|\theta_{1}\right|^{-2 \alpha-4} \theta_{2}^{4}+\left|\theta_{1}\right|^{-2 \alpha-2} \theta_{2}^{2}+\left|\theta_{1}\right|^{-2 \alpha}\right\} d \theta_{1} d \theta_{2}\right) \\
& =J J J(1)+J J J(2) .
\end{aligned}
$$

It is easy to calculate that

$$
J J J(2)= \begin{cases}O(\log N) & \text { if } \alpha=\frac{1}{2} \\ O\left(N^{1-2 \alpha}\right) & \text { if } \alpha<\frac{1}{2} \\ O(1) & \text { if } \alpha>\frac{1}{2}\end{cases}
$$


To estimate $J J J(1)$, we need the following formula which can easily be proved by integration by parts:

$$
\int_{1 / N}^{\pi} \sin ^{3}(N \theta-\pi \alpha / 2) \theta^{\nu} d \theta= \begin{cases}O\left(N^{-1}\right) & \text { if } \nu \geq 0 \\ O\left(N^{-1-\nu}\right) & \text { if } \nu \in(-1,0) .\end{cases}
$$

Obviously,

$$
J J J(1)=O\left(N^{2-3 \alpha} \int_{1 / N}^{\pi} \sin ^{3}\left\{(N+(1+\alpha) / 2) \theta_{1}-\pi \alpha / 2\right\} \theta_{1}^{1-3 \alpha} d \theta_{1}\right) .
$$

So by (22), we have

$$
\begin{gathered}
J J J(1)=O\left(N^{1-3 \alpha}\right) \quad \text { if } \alpha \in\left(-1, \frac{1}{3}\right), \\
J J J(1)=O(1) \quad \text { if } \alpha \in\left[\frac{1}{3}, \frac{2}{3}\right] .
\end{gathered}
$$

Combining (21), (23), and (24), we have

$$
\begin{aligned}
& J J J= \begin{cases}O(\log N), & \alpha=\frac{1}{2}, \\
O\left(N^{1-2 \alpha}\right), & \alpha \in\left[0, \frac{1}{2}\right), \\
O(1), & \alpha>\frac{1}{2} ;\end{cases} \\
& J J J=O\left(N^{1-3 \alpha}\right), \quad \alpha \in(-1,0) .
\end{aligned}
$$

We now estimate the term $J J$ in (19).

Clearly,

$$
\begin{aligned}
J J= & O\left(\int_{1 / N}^{\pi}\left\{\sigma_{N}^{\alpha}(\theta)\right\}^{3} d \theta_{1} \int_{1 / N}^{\pi}\left\{\sigma_{N}^{\alpha}\left(\theta_{2}\right)\right\}^{3} \theta_{2}^{4} d \theta_{2}\right) \\
& +O\left(\prod_{k=1}^{2} \int_{1 / N}^{\pi}\left\{\sigma_{N}^{\alpha}\left(\theta_{k}\right)\right\}^{3} \theta_{k}^{2} d \theta_{k}\right) \\
= & J J(1)+J J(2) .
\end{aligned}
$$

Let $\operatorname{si}(N, \theta, \alpha)=\sin \{(N+(1+\alpha) / 2) \theta-\pi \alpha / 2\}$. Using the same method used in estimating the term $J J J$, we easily obtain

$$
\begin{aligned}
\int_{1 / N}^{\pi}\left\{\sigma_{N}^{\alpha}(\theta)\right\}^{3} d \theta= & O\left(N^{-3 \alpha} \int_{1 / N}^{\pi} s i^{3}(N, \theta, \alpha) \theta^{-3(\alpha+1)} d \theta\right) \\
& +O\left(N^{-2 \alpha-1} \int_{1 / N}^{\pi} \theta^{-2 \alpha-4} d \theta\right) \\
= & O\left(N^{2}\right) . \\
\int_{1 / N}^{\pi}\left\{\sigma_{N}^{\alpha}(\theta)\right\}^{3} \theta^{4} d \theta= & O\left(N^{-3 \alpha} \int_{1 / N}^{\pi} s i^{3}(N, \theta, \alpha) \theta^{-3 \alpha+1} d \theta\right) \\
& +O\left(N^{-2 \alpha-1} \int_{1 / N}^{\pi} \theta^{-2 \alpha} d \theta\right) \\
= & \begin{cases}O\left(N^{-2} \log N\right) & \text { if } \alpha=\frac{1}{2}, \\
O\left(N^{-2 \alpha-1}\right) & \text { if } \alpha<\frac{1}{2}, \\
O\left(N^{-2}\right) & \text { if } \alpha>\frac{1}{2} .\end{cases}
\end{aligned}
$$


Equations (26) and (27) imply that

$$
J J(1)= \begin{cases}O(\log N) & \text { if } \alpha=\frac{1}{2}, \\ O\left(N^{1-2 \alpha}\right) & \text { if } \alpha \in\left(-1, \frac{1}{2}\right), \\ O(1) & \text { if } \alpha \in\left(\frac{1}{2}, \frac{2}{3}\right) .\end{cases}
$$

Similarly,

$$
\begin{aligned}
\int_{1 / N}^{\pi}\left\{\sigma_{N}^{\alpha}(\theta)\right\}^{3} \theta^{2} d \theta= & O\left(N^{-3 \alpha} \int_{1 / N}^{\pi} s i^{3}(N, \theta, \alpha) \theta^{-3 \alpha-1} d \theta\right) \\
& +O\left(N^{-2 \alpha-1} \int_{1 / N}^{\pi} \theta^{-2 \alpha-2} d \theta\right) \\
= & \begin{cases}O(1) & \text { if } \alpha \in\left[-\frac{1}{3}, \frac{2}{3}\right] \\
O\left(N^{-3 \alpha-1}\right) & \text { if } \alpha \in\left(-1,-\frac{1}{3}\right)\end{cases}
\end{aligned}
$$

Thus,

$$
J J(2)= \begin{cases}O(1) & \text { if } \alpha \in\left(-\frac{1}{3}, \frac{2}{3}\right], \\ O\left(N^{-6 \alpha-2}\right) & \text { if } \alpha \in\left(-1,-\frac{1}{3}\right] .\end{cases}
$$

Combining (20), (25), $\left(25^{\prime}\right),(28)$, and (30), we obtain that

$$
\tilde{B}_{N}^{\alpha}= \begin{cases}O(\log N) & \text { if } \alpha=\frac{1}{2} \\ O(1) & \text { if } \alpha \in\left(\frac{1}{2}, \frac{2}{3}\right] \\ O\left(N^{1-2 \alpha}\right) & \text { if } \alpha \in\left[0, \frac{1}{2}\right) \\ O\left(N^{1-3 \alpha}\right) & \text { if } \alpha \in(-1,0) .\end{cases}
$$

On the other hand we have

$$
\begin{aligned}
J_{N}^{\alpha} \geq & \iint_{0 \leq 2 \theta_{2} \leq 1 / N \leq \theta_{1} \leq \pi / 2} \prod_{k=1}^{2}\left|\sigma_{N}^{\alpha}\left(\theta_{k}\right)\right|^{3} \sin ^{4}\left(\theta_{1} / 2\right) d \theta \\
\geq & A N^{2} \int_{1 / N}^{\pi / 2}\left|\sigma_{N}^{\alpha}\left(\theta_{1}\right)\right|^{3} \sin ^{4}\left(\theta_{1} / 2\right) d \theta \\
\geq & A N^{2-3 \alpha} \int_{1 / N}^{\pi / 2}\left|\sin ^{3}\{(N+(\alpha+1) / 2) \theta-\alpha \pi / 2\}\right| \sin ^{-3 \alpha+1} \theta d \theta \\
& +O\left(N^{-2 \alpha+1} \int_{1 / N}^{\pi / 2} \theta^{-2 \alpha} d \theta\right) .
\end{aligned}
$$

Therefore, an easy computation shows that for sufficiently large $N$,

$$
J_{N}^{\alpha} \geq \begin{cases}A \log N & \text { if } \alpha=\frac{2}{3}, \\ A N^{2-3 \alpha} & \text { if } \alpha \in\left(-1, \frac{2}{3}\right) .\end{cases}
$$

Finally, from (31) and (33), we know that there is a positive constant $A$ independent of $N$ such that

$$
\int_{\mathrm{SO}(4)}\left|K_{N}^{\alpha}(V)\right| d V \geq \begin{cases}A \log N & \text { if } \alpha=\frac{2}{3} \\ A N^{2-3 \alpha} & \text { if } \alpha \in\left(\frac{1}{2}, \frac{2}{3}\right), \\ A N^{1-\alpha} & \text { if } \alpha \in\left[0, \frac{1}{2}\right), \\ A N & \text { if } \alpha \in(-1,0)\end{cases}
$$


and

$$
\int_{\mathrm{SO}(4)}\left|K_{N}^{1 / 2}(V)\right| d V \geq A N^{1 / 2} / \log N
$$

Theorem 2 is now proved.

Furthermore, we can obtain an almost everywhere convergence theorem on $\mathrm{SO}(3)$ :

Theorem 3. If $f$ is a Lebesgue integrable function on $\mathrm{SO}(3)$, then

$$
\lim _{N \rightarrow \infty}\left(K_{N}^{1 / 2} * f\right)(U)=f(U) \text { for almost all } U \in \mathrm{SO}(3) \text {. }
$$

Proof. Let $K^{*} f(U)=\sup _{N \geq 1}\left|\left(K_{N}^{1 / 2} * f\right)(U)\right|$, and let $\operatorname{Mf}(U)$ be the HardyLittlewood maximal function of $f$. If we can show $K^{*} f(U) \leq A \operatorname{Mf}(U)$ with $A$ being a constant independent of $f$, then the theorem follows easily by a standard argument (see [SW] or [B]). Checking the proof of Theorem 1, we know that

$$
K_{N}^{1 / 2} * f(U)=(\log N)^{-1} O\left(N \int_{\mathrm{SO}(3)}\left\{\sigma_{N}^{1 / 2}(\theta)\right\}^{2} f(U V) d V\right)
$$

where $V$ is conjugate to the element $S(\theta) \oplus 1$.

Let

$$
\begin{aligned}
& N \log ^{-1} N \int_{\mathrm{SO}(3)}\left\{\sigma_{N}^{1 / 2}(\theta)\right\}^{2} f(U V) d V \\
& =\log ^{-1} N\left\{\sum_{k=0}^{\log _{2}(N)} N \int_{2^{k} / N \leq d(V, I) \leq 2^{k+1} / N}+N \int_{0 \leq d(V, I) \leq 1 / N}\right\} \\
& =\log ^{-1} N I(1)+\log ^{-1} N I(2) .
\end{aligned}
$$

It is easy to see that $(\log N)^{-1}|I(2)| \leq A \mathrm{Mf}(U)$.

By Lemma 1,

$$
\begin{aligned}
& \log ^{-1} N\left|N \int_{2^{k} / N \leq d(V, I) \leq 2^{k+1} / N}\left\{\sigma_{N}^{1 / 2}(\theta)\right\}^{2} f(U V) d V\right| \\
& \quad \leq \log ^{-1} N \int_{2^{k} / N \leq d(V, I) \leq 2^{k+1} / N}|\theta|^{-3}|f(U V)| V \leq A \log ^{-1} N \operatorname{Mf}(U) .
\end{aligned}
$$

Therefore, $\left|K_{N}^{1 / 2} * f(U)\right| \leq A \operatorname{Mf}(U)$ with $A$ being a constant independent of $N$. Theorem 3 is now proved.

Recently we obtained some partial results on $\mathrm{SO}(n)$ for $n$ being greater than four. In the higher-dimensional case, computations are much more complicated than those in the cases of $n=3$ and $n=4$. So though this paper is working with $\mathrm{SO}(3)$ and $\mathrm{SO}(4)$, it clearly demonstrates how to work on the higherdimensional cases.

Finally we want to end this paper with a conjecture which is a well-known fact for $k=1$ :

Conjecture. Let $\alpha_{0}=(2 k-2) /(2 k-1)$; then for large $N$

$$
\int_{\mathrm{SO}(2 k)}\left|K_{N}^{\alpha_{0}}(V)\right| d V \cong \log N
$$




\section{REFERENCES}

[B] B. Blank, Nontangential maximal functions over compact Riemannian manifolds, Proc. Amer. Math. Soc. 103 (1988), 999-1002.

[C] E. Cartan, Sur yes domains bornés homogenes de l'espace de n variables complexes, Hamburg Univ. Math. Sem. Abhand. 11 (1936), 106-162.

[G] S. Gong, Harmonic analysis on classical groups, Springer-Verlag, Berlin and Heidelberg, and Science Press, Beijing, 1991.

[H] L. K. Hua, Harmonic analysis of functions of several complex variables in the classical domains, Transl. Math. Monographs, vol. 6, Amer. Math. Soc., Providence, RI, 1963.

[SW] E. M. Stein and G. Weiss, Introduction to Fourier analysis on Euclidean spaces, Princeton Univ. Press, Princeton, NJ, 1971.

[W] H. Weyl, The classical groups, Princeton Univ. Press, Princeton, NJ, 1939.

[Z] A. Zygmund, Trigonometric series, 2nd ed., Cambridge Univ. Press, Cambridge, 1968.

Department of Mathematical Sciences, University of Wisconsin-Milwaukee, MilwaUKEE, WISCONSIN 53201

E-mail address: fanecsd4.csd.urm.edu 